Cultures \& Conflits

09-10 | printemps-été 1993

La violence politique dans les démocraties

europénnes occidentales

\title{
La violence d'un répertoire : les sans-papiers en grève de la faim
}

Johanna Siméant

\section{OpenEdition}

\section{Journals}

Édition électronique

URL : http://journals.openedition.org/conflits/218

DOI : $10.4000 /$ conflits. 218

ISSN : $1777-5345$

Éditeur :

CCLS - Centre d'études sur les conflits lilberté et sécurité, L'Harmattan

Édition imprimée

Date de publication : 15 mai 1993

ISSN : 1157-996X

Référence électronique

Johanna Siméant, «La violence d'un répertoire : les sans-papiers en grève de la faim », Cultures \&

Conflits [En ligne], 09-10 | printemps-été 1993, mis en ligne le 04 mars 2005, consulté le 30 mars 2021. URL : http://journals.openedition.org/conflits/218; DOI : https://doi.org/10.4000/conflits.218

Ce document a été généré automatiquement le 30 mars 2021.

Creative Commons License 


\title{
La violence d'un répertoire : les sans-papiers en grève de la faim
}

\author{
Johanna Siméant
}

1 "Alors on a choisi de se torturer nous pour leur faire mal à eux." ${ }^{1}$

2 Considérer les grèves de la faim à partir des catégories de la violence politique protestataire, revient à souligner l'intérêt que représentent les phénomènes de "violence contre soi" rapportés à la problématique du marquage des corps, à celle des "technologies politiques du corps"2, envisagées non plus du point de vue d'une entité politique dominante, où "marquages" et "disciplines" sont autant d'armes au sein des arsenaux étatiques, mais plutôt sous l'angle du sujet politique. De même que l'étude de la manifestation comme répertoire ${ }^{3}$ est récente ${ }^{4}$, de même le caractère souvent considéré comme exceptionnel des grèves de la faim - quand il n'est pas explicitement renvoyé à des catégories religieuses plutôt que politiques ${ }^{5}$ ne doit pas faire négliger la profusion des grèves de la faim dans la deuxième moitié du XXème siècle. Depuis le début des années 70, la grève de la faim semble être à certaines catégories d'acteurs (et notamment aux mouvements et protestations politiques issus de, ou liés à, l'immigration) ce que la grève fut aux mouvements ouvriers du début du siècle. Charles Tilly soulignait qu'il nous manquait "une analyse des transformations des modes d'action populaires"6. La dichotomie, instituée au coeur de la sociologie politique, entre modèles d'action collective et modèles d'action individuelle peut en partie expliquer ce faible intérêt pour la question ${ }^{7}$. Or considérer la grève de la faim comme un répertoire permet de ne pas se limiter à l'étude des dimensions instrumentales du "procédé". Le répertoire suppose certes un univers de rareté des moyens ${ }^{8}$, mais aussi un univers de sens partagé, préalable à l'action. De même que de nombreux groupes sont constitués autour de Causes qui sont au principe de leur mobilisation, les répertoires portent la marque des agents qui y ont recours, en même temps qu'ils les constituent. Sous la dénomination "grève de la faim" se cachent ainsi de multiples façons de "se faire violence" au nom de violences déjà subies. Aussi, il semble essentiel de ne pas séparer l'étude de la grève de la faim de celle des groupes qui s'approprient ce répertoire : par 
conséquent, nous étudierons plus particulièrement une catégorie spécifique de grèves de la faim : celles des sans-papiers en France ${ }^{9}$.

\section{LE RECOURS DES SANS-PAPIERS A LA GREVE DE LA FAIM}

Les deux plus grandes vagues de grèves de la faim ayant eu lieu en France hors milieu carcéral sont celles de 1972-1973 et de 1991-1992. Dans les deux cas il s'agissait de grèves de sans-papiers : étrangers sans titre de travail dans le premier cas, déboutés du droit d'asile dans le second, avec également de nombreux cas de personnes touchées par la double peine. Ce constat, ajouté à celui de la persistance du recours à la grève de la faim par des immigrés, sans-papiers ou non, fait de la grève de la faim un véritable répertoire de ce groupe social aux contours flous, unifié seulement par sa situation ambivalente voire son rapport inexistant à la nationalité et à la légalité : il s'agit, dans tous les cas, de personnes en situation illégale ou en passe de le devenir. Les grèves de la faim des déboutés et celle du mouvement contre la double peine ont une physionomie assez différente. Celle de janvier-mars 1992 est la troisième grève de déboutés du droit d'asile, sur Paris. Elle s'insère dans la longue liste de grèves qui a marqué l'année 1991 et le premier semestre 1992. On a compté environ 1500 déboutés en grève sur cette période, soit le plus gros nombre de grévistes hors milieu carcéral jamais enregistré en France dans une grève collective. Les grèves se sont succédées dans toute la France, notamment par le biais du bouche à oreille au sein de la communauté kurde-turque. Leurs premiers objectifs étaient d'obtenir des papiers ou d'éviter l'expulsion et, pour les associations de soutien, de dénoncer les méthodes de l'OFPRA $^{10}$ ainsi que la situation des déboutés. Les associations françaises (Fasti, Gisti, Cimade, MRAP...) et "communautaires" (AISOHAF, CAIF,...), mises devant le fait accompli des grèves, ont procédé à une mise en forme politique de ces dernières, tentant de souligner la situation des déboutés du droit d'asile en France. Toute une partie de leur travail a ainsi contribué à donner aux grèves l'allure d'un petit "mouvement de masse". Depuis la parution de la circulaire du 23 juillet 1991, qui s'est révélée décevante pour les milieux associatifs en attente d'un traitement global de la question des déboutés, et l'arrivée de Paul Quilès au ministère de l'intérieur, une plus grande fermeté des pouvoirs publics est de mise à l'égard des grèves : ce qui, joint à la lassitude de militants associatifs engagés dans le soutien à ces grèves depuis un an et demi et au faible nombre de régularisations obtenu, explique en grande partie l'essoufflement du mouvement. Les grèves les plus récentes (avril à juillet 1992) ont vu l'intervention de la police mettre un terme au mouvement et parfois procéder à des expulsions. La troisième grève des déboutés du droit d'asile est apparue à Paris comme une lutte quasi clandestine : pas de liste de noms de grévistes, les Français ne sachant pas toujours qui ils défendaient; des associatifs considérant le recours à la grève de la faim comme profondément sans issue, avaient d'ailleurs essayé d'en dissuader les grévistes. Sans succès puisque les déboutés, hébergés depuis un an dans la salle paroissiale où s'étaient déroulées les deux premières grèves de Paris, les mirent devant le fait accompli de cette troisième grève. Lors de la grève de la faim relative à la double peine deux autres grèves se sont tenues à Lyon et à Lille. Le comité contre la double peine avait déjà montré un savoir-faire certain dans la spectacularisation de ses actions. Cette grève fut beaucoup plus "concertée" que celle des déboutés, ainsi qu'en 
témoigne cet extrait d'entretien: "Le comité voulait faire une action collective, on savait pas laquelle. Ils étaient débordés de dossiers [de personnes expulsées ou en voie de l'être]... 1300 dossiers... Il fallait faire une épreuve de force... Parmi eux, il y avait Momo qui avait déjà fait une grève de la faim... En parlant on avait dit pourquoi pas un sit-in? Comme ils vont pas céder tout de suite, il faut une action longue. Comme on était tous taulards, il y avait déjà moi qui avais fait une grève de la faim, et puis Momo, trois ou quatre personnes... Quand il y a eu le meeting, l'idée de la grève de la faim avait germé... Au meeting je tenais le stand grève de la faim... Il y avait un panneau appelant les gens à faire la grève, il y avait déjà mon nom en haut en premier pour montrer qu'il $\mathrm{y}$ avait déjà quelqu'un. Il y a plein de gens qui se sont inscrits... des gens qui voulaient faire des grèves de soutien de deux jours... Y'avait que des gens touchés... Y'a des nanas qui ont fait des jeûnes de soutien..." ${ }^{11}$. Certains traits sont communs aux grèves des déboutés et de la double peine, voire plus généralement aux grèves de sans-papiers. Tout d'abord leur longueur: une soixantaine de jours pour la troisième grève des déboutés sur Paris, une cinquantaine de jours dans le cas de la double peine. Le chiffre de quarante jours est souvent atteint sur les autres grèves. Cette durée est permise en partie par le recours à la "grève au thé sucré" - pratique turque que de nombreux grévistes ont adoptée -, aux vitamines et, dans certains cas, à l'absorption de yaourt liquide, ou de bonbons... Elle est également due au caractère de masse de ces grèves, qui rendait pour les préfectures et le gouvernement un règlement plus difficile et plus long sans le recours à des mesures globales qu'ils tenaient à éviter. S'y ajoute le contexte de campagne électorale qui interdisait au gouvernement tout geste susceptible d'être perçu comme trop favorable à l'égard d'une catégorie aussi illégitime que celle d'étrangers clandestins, demandeurs d'asile ou "délinquants". La grève des déboutés à Paris s'est ainsi conclue le 23 mars 1992, soit un jour après les élections régionales et cantonales. Un autre trait notable de ces grèves est leur caractère de groupe : c'est une caractéristique typique des grèves de sans-papiers, qu'on retrouve rarement ailleurs. Les grèves de 35 ou 40 personnes ont été nombreuses ${ }^{12}$. On a compté simultanément en France jusqu'à 400 déboutés grévistes de la faim. Enfin, une troisième caractéristique est le flou qui règne autour du nombre exact de grévistes. Les grèves collectives des sans-papiers sont très souvent évaluées à 5 ou 10 personnes près : ainsi, tous les soirs quelques clandestins sans logement arrivaient dans la salle paroissiale, où la grève des déboutés se déroulait, et dormaient dans l'église, parmi les grévistes, sans que les observateurs extérieurs et les associations de soutien ne sachent toujours si elles avaient affaire à des grévistes ou à des personnes de passage. La grève de la faim de la double peine, qui fut pourtant beaucoup plus individualisée (le premier numéro du Journal de la grève ${ }^{13}$ présenta les noms de tous les grévistes accompagné de leurs photos) vit le nombre des grévistes, 19 au départ, aller augmentant puis décroissant, suite à des abandons ${ }^{14}$, sans que l'on en connaisse exactement le nombre final. Et si la technique du tableau indicateur à l'entrée du local semblait mieux maîtrisée, le tableau finit surchargé de ratures, illisible et abandonné à son triste sort, comme si la grève comptait plus que les grévistes qui la composaient, dans un effort d'agrégation de répertoires individuels en une action collective ${ }^{15}$.

\section{STRATEGIES DE LA GREVE DE LA FAIM}


6 Le parallélisme est presque parfait entre la menace du recours à la violence en général et la menace du recours à la violence contre soi. L'étude que fait Schelling ${ }^{16}$ des situations rattachées au pouvoir de se lier soi même, "le pouvoir des faibles", convient ainsi particulièrement à l'analyse de la grève de la faim. Les grévistes, quittant leur situation de clandestins, se dévoilent radicalement et tentent de se lier eux-mêmes par une menace de mort, de rendre leur conduite et celle de leurs alliés parfaitement prévisible, avec l'espoir que cette dernière sera suffisante pour faire reculer les pouvoirs publics et obtenir des papiers. Cette façon de se lier soi-même est d'autant plus explicite qu'aux yeux des déboutés du droit d'asile, notamment kurdes-turcs, le fait de mener une protestation publique en France interdit véritablement de rentrer sans danger au pays, quels qu'aient été les risques antérieurs de le faire. L'expulsion devient alors véritablement inenvisageable pour eux, ayant modifié leur situation antérieure au point qu'on ne saurait désormais effectuer de rapatriement forcé sans risques - et qu'eux mêmes sont prêts à se battre encore plus farouchement afin d'éviter leur expulsion. En tant qu'acteurs sociaux dépourvus d'accès aux centres de pouvoir, les sans-papiers utilisent logiquement la gamme peu étendue de moyens dont ils disposent pour se faire entendre. Ce n'est pas un hasard si le recours à la violence contre autrui est profondément interdit à des étrangers clandestins demandant la reconnaissance d'un statut au sein d'une communauté politique. A l'inverse les agriculteurs peuvent se permettre des violences dont l'exercice par des clandestins serait considéré comme inadmissible. Cette éventualité d'un recours à la violence, interdit en réalité par le rapport de force, était souvent évoquée lors de la grève de la double peine, sans qu'il faille y voir seulement un argument destiné à impressionner l'adversaire ou à s'attirer les faveurs de militants peu favorables à la non-violence. Ainsi, lors d'une conférence de presse, un gréviste évoquera un ensemble de moyens allant de la grève de la faim à la lutte armée en passant par la violence dans les banlieues ou les grèves de la faim assorties d'occupation de locaux : "Les Français, le peuple français ils vont le subir. Pas les ministres dans leur bureau. Les mômes dans les banlieues ils ont une violence ils ont une haine.... Le phénomène américain c'est nous qui allons le déclencher sans le vouloir. C'est même plus qu'il y en a marre c'est qu'il y en a marre d'en avoir marre.... Y'a un racisme qui commence à naître un racisme du commerçant français ${ }^{17}$ un racisme de.... $Y^{\prime}$ a un discours violent à force. [...] On est prêt à prendre cent volontaires à faire une grève de la faim dans une télé à les forcer à parler de nous qu'enfin ils le fassent parce que là c'est prouvé c'est la censure gouvernementale. De toute façon moi je suis pas contre la lutte armée si la cause est juste"18. Dans son étude sur le mouvement des droits civiques aux USA, des années 50 aux années 70, Oberschall ${ }^{19}$ expliquait le contraste entre les mouvements du Nord, plus violents, et ceux du Sud, plus pacifiques, par le ratio risques / avantages et la structure sociale des collectivités concernées. La communauté noire du nord était segmentée et prolétarisée, celle du sud bénéficiait de l'appui du clergé protestant, de l'administration fédérale et des élites blanches du nord: autant de supports qu'elle ne pouvait se permettre de perdre par une attitude violente. On est tenté de faire ici le rapprochement avec la situation de déboutés et d'expulsés qui ne pouvaient se permettre de perdre, par une attitude violente, les rares soutiens dont ils disposaient celui d'associations (la plupart du temps chrétiennes ou liées aux mouvements non violents comme la Cimade, la Pastorale des Migrants, etc.), d'aide aux réfugiés et aux immigrés; il leur fallait procéder à une instrumentalisation de l'Eglise. Il est ainsi courant que les grévistes s'imposent dans une église et mettent les autorités 
ecclésiastiques devant le fait accompli, avec succès la plupart du temps ${ }^{20}$. Les membres du comité contre la double peine avaient quant à eux prévenu les responsables de la Cimade que de gré ou de force ils s'installeraient dans leurs locaux. Par ailleurs la pratique de la grève de la faim dans les églises permet une protestation sans trop grand danger d'arrestation, déterritorialisant en quelque sorte un mouvement public de clandestins. Ainsi, la grève de la faim a joué comme un répertoire qui permettait en soi de mobiliser des soutiens et des ralliements, l'Eglise ne pouvant que soutenir ou, au pire, tolérer un moyen aussi pacifique. Par ailleurs la référence gandhienne dans les milieux catholiques et non violents où de nombreux militants ont une pratique sinon de la grève de la faim au moins du jeûne de protestation, permettait de donner à ces grèves une dimension qui n'était pas toujours celle que leur attribuaient les grévistes. Se distinguent alors deux représentations de la grève de la faim : l'une rapportée à une conception du monde, pratiquée par des chrétiens, l'autre considérée comme seul moyen disponible. Cela souligne l'ambiguïté fondamentale des grèves de la faim, "violence soutenue par des non violents" - et parfois pratiquée par d'"anciens violents" : [Vous avez déjà fait des grèves ?] Oui une grève à Orléans en 1989 pour soutenir un Zaïrois débouté dans des conditions révoltantes. Avant j'avais fait des grèves de 4 jours, 6 jours. Comme moyen. Je suis pas partisan des grèves illimitées. Pour le zaïrois on avait décidé de faire la grève de la faim 15 jours c'était un moyen non violent.... La grève de la faim est un moyen non violent mais... peut être pas comme ils la font eux [les Kurdes]... Y'a des références à Gandhi pour faire appel à la conscience des gens... [...] donner un poids fort... qu'on se contente pas de signer une pétition..." ${ }^{21}$. Il existe toute une rhétorique de la grève de la faim qui tourne autour de la menace de lésions irrémédiables, voire de la mort. D'un point de vue stratégique, la menace d'une violence irrémédiable faite à soi-même est le point central de la grève de la faim, le recours à cette dernière fonctionnant sur le danger d'une modification radicale de la grille de conflit: la mort d'un gréviste ou un coma. Si, à l'égard de "terroristes", l'indifférence permet de répondre à ce défi comme le fit le gouvernement anglais à l'égard des grévistes irlandais, peu de gouvernements démocratiques sont prêts à prendre la responsabilité de la mort d'un homme n'ayant pas eu, lui même, recours à la violence. La menace de "séquelles irréversibles" est ainsi utilisée chez les deux parties en présence : pouvoirs publics et comités de soutien aux grévistes, afin d'imputer à l'autre la responsabilité de la souffrance des grévistes. Lors des grèves de déboutés, les autorités tentaient de disqualifier des associations dénoncées comme irresponsables car apportant leur soutien à des entreprises suicidaires. Les responsables associatifs sont quant à eux partagés entre l'utilisation de la menace de séquelles irréversibles, et l'inquiétude à l'idée d'être responsables de morts ou de comas. Cette position inconfortable est illustrée par les propos d'un responsable associatif: "Au début la grève c'est un atout, après c'est un handicap dans les négociations quand tu es responsable des grévistes". En témoignent ces discussions, une semaine avant la fin de la grève des déboutés, sur l'attitude à adopter : "On est dans la seule période où les pouvoirs publics ont plus peur que les grévistes. [...] Les médecins ne sont pas affolés. [...] Il est très important que les pouvoirs publics flippent. Quand je vois des grévistes qui sont capables de passer deux heures dans la fumée [de cette réunion] je suis pas inquiet. On les trahit complètement au dernier moment où on peut être ferme. Quand j'entends négociation je trouve que c'est de la trahison"22. "A l'époque de la première grève on a paniqué aussi quand il y a eu une ouverture. [...] On est tombé dans le piège parce qu'on a paniqué sur la santé des gens. C'est pas ici qu'on doit paniquer. En face ils 
cherchent une issue il faut que les grévistes s'en sortent mieux qu'eux. Je me sens très mal à l'aise mais j'essaie de ne pas paniquer. Il ne faut pas de négociations"23. Il s'agit de donner le mauvais rôle aux pouvoirs publics : qu'ils nourrissent de force des grévistes ou qu'à l'inverse ils laissent survenir des comas. Tout se joue alors sur le fait de convaincre les pouvoirs publics de la détermination des grévistes. Aussi l'instrumentalisation par les deux parties de tout ce qui a trait à l'état de santé des grévistes (dossiers médicaux, déclarations des médecins...) revêt une importance considérable. L'utilisation de l'expertise médicale est un trait incontournable: la reproduction dans les communiqués de presse de certificats médicaux faisant état, selon une formule classique, de "lésions imminentes", est une constante des grèves de la faim. Il est par ailleurs fréquent que des médecins sympathisants émettent des communiqués alarmistes, ou au cours de conférences de presse, accompagnent leurs diagnostics d'opinions franchement engagées, posant ces dernières dans la suite logique des autres, comme le fit le médecin des grévistes de la double peine, soulignant lors d'une conférence de presse que: "Quand des hommes décident cela, il faut se demander qui sont les responsables": ce sont "les gens qui tiennent un double langage", ce sont ceux qui utilisent des méthodes qui lui "rappellent la période de Vichy", qu'il a vécue. Il est très applaudi ${ }^{24}$. A l'inverse, les médecins les plus rodés aux grèves de la faim, souvent pour avoir longuement exercé en milieu pénitentiaire, ont une attitude moins alarmiste à l'égard des grèves et se cantonnent dans leur rôle d'expertise, qu'ils justifient au nom d'une crédibilité dont il soulignent l'intérêt stratégique : "On peut pas avoir deux casquettes : celle de militant et celle de docteur ; on n'est plus crédible par rapport aux pouvoirs publics et quand on écrit aux ministères et que, deux jours avant on poussait un coup de gueule dans Libé, on vous croit plus. On a dit aux grévistes : demandez pas qu'on fasse des communiqués dans la presse. C'est pour ça qu'on a refusé d'aller à la conférence de presse. Du coup la seule lettre qu'on ait faite au ministère des affaires sociales qui est notre supérieur hiérarchique pour dire là vous avez des gens dans des états graves, a eu de l'effet : quarante-huit heures après, on a eu un coup de fil très inquiet de la préfecture. Ils voulaient à tout prix éviter les morts"25. De même, la recherche de données fiables sur l'état des grévistes, qui permette dans le cas d'une grève "peu sérieuse" de refuser des négociations sans craindre de comas, amène les pouvoirs publics à chercher le plus de renseignements possibles sur l'état des grévistes, bien sûr par les Renseignements Généraux mais aussi par d'autres moyens, plus ou moins discrets, comme par exemple la proposition faite à une association de médecins en charge de la grève des déboutés d'introduire discrètement "un médecin de la préfecture" parmi eux ${ }^{26}$. Le refus des grévistes de se faire examiner est souvent considéré comme une preuve de la faible gravité de la grève. A l'inverse, il est arrivé que des comités de soutien demandent aux grévistes d'arrêter une grève tant la présence de "tricheurs" au sein de la grève décrédibilisait cette dernière aux yeux des pouvoirs publics, persuadés que "tous les grévistes mangeaient", et mettait par conséquent en danger la vie des "vrais" grévistes. Enfin, si la grève n'obtient pas de résultat, les pouvoirs publics étant peu convaincus que les grévistes aient vraiment l'intention "d'aller jusqu'au bout", ces derniers ont souvent recours à une technique telle que le passage à la grève de la soif, l'arrêt de la prise de vitamines, de médicaments ou de thé sucré27. C'est là qu'il s'agit de brûler son dernier vaisseau, gardé précieusement en réserve: d'où toutes les techniques préalables visant à prolonger la durée de la grève sans dommages irréversibles. Les grévistes savent que "l'on ne meurt pas en France d'une grève de la faim" ${ }^{28}$. Ce qu'il leur faut "réussir à 
risquer", ce sont ces séquelles irréversibles décrites par des docteurs dans les conférences de presse ou les tracts distribués ${ }^{29}$. La grève de la faim au sucre et surtout aux vitamines permet ainsi de garder encore un atout dans sa manche, dans une configuration où les grévistes paraissent ne plus pouvoir continuer dans l'escalade en s'étant engagés complètement, sans résultat. Le dénouement des grèves passe fréquemment par une crise où les grévistes entament une grève de la soif ou arrêtent de prendre leurs vitamines. Les résultats ne se font pas attendre: hospitalisations massives voire, plus rarement, comas. Il est rare que ne s'ouvrent pas alors des négociations avec les préfectures, lesquelles accordent en général quelques concessions par rapport à leurs propositions initiales. Un point central de l'organisation des grèves de la faim en groupe repose sur le contrôle de la grève, l'interdiction de défection, afin d'empêcher que la grève ne finisse en perdant un à un ses grévistes. Ce contrôle eut, chez les déboutés de Paris, une dimension très explicite: les grévistes s'étaient barricadés dans la salle paroissiale afin d'empêcher l'abandon de la grève par certains d'entre eux. Le comité de soutien insista pour faire ouvrir les portes peu après, mais cette technique en dit long sur les manières parfois expéditives de coller à un répertoire considéré comme non violent. De fait, le contrôle mutuel exercé par des grévistes enfermés ensemble 24 heures sur 24 suffit en général à éviter des défections, d'autant qu'il s'agit de convaincre le comité de soutien que l'on est prêt à mourir. Le comité étant chargé des négociations, il est bon qu'il soit lui même convaincu de la situation désespérée des grévistes, voire paniqué de façon assez "crédible" à l'égard des autorités. Les seuls abandons le sont donc pour "raisons médicales" : argument qui recouvre en fait un état de santé ne permettant pas de continuer la grève sans risque de mort ou d'apparition prématurée de ces lésions irréversibles ${ }^{30}$ qu'il faut garder en réserve, et non "gaspiller" avant la phase finale de la grève. L'influence exercée sur les comités de soutien, chargés des négociations ${ }^{31}$ et de la maintenance politique de la grève, est une dimension constitutive de cette dernière. Les grévistes passent une grande part de leur temps à dire aux membres des comités de soutien qu'ils iront "jusqu'au bout", même si cela implique d'aller "s'immoler devant la préfecture ou le ministère de l'intérieur: " Vous prévenez les télévisions, on prend un bidon d'essence et on se brûle devant l'Eglise Saint Joseph, vous prévenez les télés et on le fait, s'il faut ça, qu'ils aient un mort pour que les journaux en parlent, alors peut-être qu'il y aura les papiers, de toute façon on continue ${ }^{32}$. La grève de la faim ne peut toutefois être réduite à ces seules dimensions stratégiques. On a souvent souligné les dimensions de la culture irlandaise qui pouvaient expliquer les grèves de la faim des Irlandais de l'IRA: une mythologie sacrificielle ancienne, réactivée par les Pâques sanglantes de 1916, la référence au martyre et, notamment, à celui des fondateurs de la République ${ }^{33}$. Si le recours aujourd'hui répandu aux grèves de la faim oblige à infirmer certaines explications culturalistes vouées à la tautologie, il n'en reste pas moins que plusieurs caractéristiques objectives des groupes ayant recours à la grève de la faim paraissent être en mesure d'expliquer la réussite et les modes d'importation de ce répertoire chez les sans-papiers.

\section{UNE TECHNIQUE POLITIQUE DES CORPS}

La grève de la faim est un répertoire qui ne fait sens que parce qu'il s'insère dans une 
anthropologie politique du corps qui touche à la fois à la question du monopole de la violence légitime et aux dimensions symboliques de la violence physique. Ce que la grève de la faim et le suicide politique mettent en jeu, c'est, pour paraphraser Max Weber, le monopole de la violence légitime sur les corps, ce qui permet ou non "le droit à disposer de son propre corps" : "Madame, en France il y a des lois, on ne se suicide pas comme ça" (Un sous-préfet à une militante d'un comité de soutien de Creil-Montataire, qui l'avertissait de nuit, par téléphone, de l'imminence d'une tentative d'immolation d'un des grévistes de la faim). Il est à cet égard significatif que les pratiques de gavage de force aient longtemps existé à l'égard des grévistes de la faim ${ }^{34}$. De même, on a pu assister, lors de plusieurs grèves de déboutés, à des interventions de la police, accompagnée de médecins, sur les lieux de grève, voire à des hospitalisations de force qui posaient très réellement le problème du droit à se laisser mourir ${ }^{35}$ ou qui pour le moins, prenaient ce prétexte comme argument afin de faire cesser la grève. Si avec Michel Foucault on considère comme pertinente la problématique du marquage des corps, si avec Georges Balandier on admet que le corps est un support du marquage politique, un opérateur politique ${ }^{36}$, alors on doit en corollaire poser comme possible l'appropriation de ce support par des acteurs ayant un accès limité aux autres formes de protestations : ni le droit de vote ni le droit à la manifestation ${ }^{37}$. Faire souffrir son corps en imputant cette violence au "pouvoir d'en face", c'est retourner contre ce dernier ce qu'il a pratiqué ou est susceptible de pratiquer légitimement contre soi (emprisonnement, expulsion, coups) et remet profondément en cause le monopole auquel il est susceptible de prétendre. Ainsi les grèves de la faim remettaient aussi bien en cause les pouvoirs publics français, qui se voyaient imputer la responsabilité de corps souffrants sans existence politique ni juridique ${ }^{38}$, que les partis turcs et kurdes, (TKPML, Dev Sol, PKK...), dont le contrôle souvent violent ${ }^{39}$ sur les Kurdes et Turcs et les capacités de mobilisation est un fait avéré. Les dirigeants de ces partis perdaient le contrôle qu'ils exerçaient, au point de faire remarquer aux grévistes que ce n'était pas en France mais en Turquie qu'il fallait faire la grève de la faim et se révolter. Une explication du recours fréquent à la grève de la faim des sans-papiers peut être avancée à partir des parcours des deux populations évoquées ici. Ce qui caractérise ces grévistes dans le rapport politique qu'ils sont susceptibles d'entretenir à leur corps, c'est précisément le rapport de domination et de violence que ces corps ont subi, cette incorporation de la domination qu'a constitué un marquage symbolique et physique des corps. Il est ainsi frappant, dans le cas des grèves que nous avons pu observer, que les groupes qui y ont recours sont, dans les deux cas, passés par ce que nous qualifierons d'"objectivation politique des corps" : prison, mauvais traitements, torture, infligés par des représentants de l'appareil étatique. Ces expériences sont des clefs susceptibles d'expliquer comment certains stigmates ont été retournés dans l'action ${ }^{40}$, comme si ces sans-papiers étaient passés par une série de réductions de leur propre personne, du statut de sujet politique à leur seule dimension physique de corps. Ainsi, la première caractéristique des personnes touchées par la double peine est qu'elles sont toutes passées par la prison. Or, par la violence physique permanente qu'il suppose, le milieu carcéral favorise des pratiques de violence, contre les autres mais aussi contre soi, au point que grèves de la faim et mutilations semblent n'être qu'une façon de se réapproprier un corps soumis aux disciplines et au panoptique ${ }^{41}$ carcéral, à l'absorption massive de tranquillisants prescrits avec prodigalité, prolongeant souvent une toxicomanie antérieure ${ }^{42}$, aux fouilles corporelles dont on connait la méticulosité, etc. Tous les grévistes que nous avons rencontrés avaient un état de santé ou, au moins, 
un aspect propre aux anciens détenus et/ou aux anciens toxicomanes : dentition (très) abîmée, calvities précoces, etc., et ce d'autant que la situation de clandestin a pour corollaire l'impossibilité d'accéder avec facilité à des soins médicaux qui nécessitent des papiers tels que carte de sécurité sociale, mutuelle, etc. Qu'il s'agisse de tatouages (plusieurs grévistes de la double peine en portaient), d'automutilations (là encore un répertoire propre aux prisons), de grèves de la faim, cela passe par toute une série de techniques du corps qui éclairent la violence faite à soi comme réponse à ce qui est vécu comme la violence de l'univers carcéral. Beaucoup de grévistes avaient déjà une expérience de la grève de la faim en prison, et certains grévistes de la double peine situent l'origine de leur engagement dans le passage par la prison, soulignant que la domination des corps a permis la découverte et la maitrise de cette violence contre soi en même temps qu'elle a parfois abouti à une violence plus classique, du type de la mutinerie : "J'étais de la mutinerie de Bois d'Arcy, où il y avait même pas le cubage d'air légal par cellule. [...] J'étais partisan de l'automutilation. Se couper le petit doigt... Moi personnellement j'ai reproduit des trucs que j'ai appris en prison. Mon frère il était à Fleury il a fait partie du mouvement de ceux qui se sont coupés un petit doigt avec Knobelpiess... Quand tu vis dans un milieu dur comme la prison qui est hors de tout, y'a pas beaucoup de moyens... ou alors t'as les moyens de t'évader. [...] En taule j'ai fait un mois et trois jours euh oui trente six jours de grève [...] on m'a mis à l'isolement à la limite c'était mieux ça m'a permis de me ramasser [...] Souvent ils utilisent des méthodes qui ne font que te renforcer [...] J'ai appris plein de choses en prison j'ai appris à bouquiner, à écrire ; enfin je savais écrire mais à écrire ce que je pense. C'est un milieu criminogène tu rencontres plein de gens, t'apprends tout ce que t'avais pas à apprendre". (Il montre aussi les cicatrices assez nettes sur ses bras et poignets des mutilations qu'il s'est faites en prison.) ${ }^{43}$. Mais la prison est aussi évoquée comme un laboratoire politique, une réorientation dans la "carrière morale" ${ }^{44} \mathrm{du}$ délinquant qui décide, avec succès ou non, "d'abandonner la violence" et mobilise en tout cas ce passage en prison au principe de son recours à la grève de la faim : "Au début ça a commencé par autre chose que la double peine. Momo pendant la prison a beaucoup bouquiné. On lit beaucoup en prison. Lui la délinquance c'est fini. On décide que c'est fini on est clair. [...] J'ai déjà fait une grève de la faim je suis allé quatre fois en prison. Huit ans de prison alors que j'étais innocent. J'avais perdu 21 kilos. J'ai quelques séquelles physiques et psychiques ${ }^{45}$. Les grévistes déboutés du droit d'asile demandent quant à eux le statut de réfugiés politiques au nom de persécutions dont ils s'affirment victimes dans leur pays d'origine. S'étant vus refuser le statut de réfugiés en raison de "preuves insuffisantes", il n'en reste pas moins que beaucoup sont effectivement passés par la prison, ne serait-ce "qu'un" mois ou deux, voire y ont déjà pratiqué des grèves de la faim et subi la torture. L'administration de la preuve des tortures subies est une étape cruciale dans le parcours du demandeur d'asile, le certificat médical constituant une pièce de poids dans un dossier. Or les examens médicaux, pratiqués longtemps après ces tortures, ne peuvent déceler que des cicatrices extérieures marquées - traces que tout tortionnaire averti tente d'éviter. Cependant l'OFPRA prend rarement en considération les récits de torture des réfugiés si ces derniers ne sont pas à même de fournir un certificat "crédible". Ainsi, la parole des grévistes ne vaut pas tant que les traces que leur corps serait susceptible de révéler : comme dans la grève de la faim, c'est la souffrance visible des grévistes, comme cicatrice ou comme souffrance publique attestée par un système fait d'hospitalisations, de déclarations de médecins et de maigreur visible et publique, qui est finalement plus opératoire que leur parole. Mais 
outre le fait que tous les grévistes de la faim n'ont pas été torturés ou incarcérés, la domination incorporée ne se limite pas à ce type de traitement des corps. Abdelmalek Sayad soulignait combien la représentation de l'immigré comme doublement absent de l'ordre politique, a comme point extrême sa réduction à son corps biologique et à son statut de force de travail ${ }^{46}$. Très souvent, aussi bien chez les militants associatifs que chez les grévistes, on constate ce sentiment selon lequel il n'y a que la menace de mort qui soit susceptible d'attirer l'attention des pouvoirs publics à leur égard : "Notre grève était partie pour un truc dur qui était : pas s'arrêter à la première promesse ; un truc dur qui se voit leur dire voyez on mange pas une forme de hurlement de gens qui vont entendre... Quand tu fais une grève comme ça tu sens qu'en face t'es rien mais tu pèses du poids t'as pas d'autre moyen. Ils s'en foutent" ${ }^{14}$. Cette objectivation politique du corps du clandestin, soumis au risque d'un contrôle d'identité au "faciès" qui, en révélant sa situation aurait comme conséquence l'expulsion ${ }^{48}$, est liée, comme le rappelle Sayad, à une représentation de l'immigré selon laquelle ce dernier ne saurait avoir ni famille ni bagage puisqu'il n'est qu'un corps/force de travail : constitué par la loi en corps uniquement - et corps qu'il s'agit de détecter puisqu'il ne fait pas partie de l'ordre national -, le débouté, le clandestin gréviste fait de son statut de corps biologique une ressource : en devenant un corps publiquement souffrant - mais de son fait. Tout se passe alors comme s'il s'agissait de jouer "les droits de l'homme" - et de son corps -, plutôt que ceux du citoyen, catégorie dont les clandestins sont par définition exclus. Comment s'étonner alors que des grévistes kurdes ayant déjà mené en mai 1991 des grèves de solidarité avec les Kurdes d'Irak expliquent que "c'était différent, c'était politique. Là, c'est humanitaire" ? Reconquérir ce qui est vécu comme une identité, c'est-à-dire avant tout une existence publique, reconquérir le statut de sujet politique à partir du seul support qui reste quand on est précisément privé de corps politique : il y a dans le recours à la grève de la faim une dimension de l'affirmation de soi comme sujet politique qui pose la question du corps politique ${ }^{49}$ des grévistes. "Pendant tout ce temps là ils avaient une identité... du jour où ils ont attaqué la grève de la faim ils n'avaient plus peur ils avaient leur nom çà je l'ai super ressenti après dix-sept ans en clandestinité... avec les faux papiers.... t'arrêtes de te cacher tu dis je suis là je m'appelle comme ça. [...] La fin a été la plus dure. Arrêter la grève ça a été beaucoup plus dur que faire la grève... C'était un truc hyper fort... Des fois je me demande si je suis pas resté à travailler au comité [contre la double peine] pour ça. [...] J'étais venu au comité pour mon cas à moi j'avais pris la rage au bout d'un certain temps à force de prendre des coups j'avais voulu faire face et j'ai fait face comme un gosse qui dit "ah tu m'as fait ça" une sorte d'acceptation en même temps tu veux te battre, c'était presque enfantin" ${ }^{10}$. La grève de la double peine (qui concerne des étrangers délinquants), fut ainsi l'occasion pour beaucoup de grévistes d'un apprentissage politique et, plus encore, d'une redéfinition de leur image publique, passant du statut de suspects à celui de victimes, montrant leur capacité à user d'un répertoire non violent alors même qu'ils étaient accusés de violences, ou considérés comme susceptibles d'y avoir recours (qu'on se souvienne de la grève de la faim de Christian Delorme et de neuf jeunes "casseurs" qu'il avait emmenés voir un film sur Gandhi avant de leur proposer un jeûne de protestation, au moment des émeutes des Minguettes en 1983). Des grévistes se sont retrouvés auréolés d'une autorité morale qui constituait la symétrie parfaite de leur statut antérieur de "violents" : délinquants, vendeurs de drogue, braqueurs et proxénètes ${ }^{51}$. On comprend alors que certains grévistes se soient attachés à ce statut au point de vouloir continuer cette grève dans 
l'affirmation de soi, sans plus grande considération stratégique. Tous avaient en quelque sorte retrouvé leur "full size"52, accédé à une grandeur avec laquelle leurs amis mêmes ne les avaient jamais considérés : "J'ai des gens à qui je la dois cette grève de la faim. Je partirai pas comme ça, moi je veux un papier pour montrer à mon fils que son père il a bien fait la grève de la faim, parce que c'est vrai que même un bon copain à moi il m'a dit allez Hocine arrête je sais bien que tu la fais pas la grève. Je veux finir en beauté. Moi je suis content j'ai appris beaucoup de choses ici"53.

\section{AU NOM DE QUOI SE FAIRE VIOLENCE?}

Un répertoire est approprié selon des savoirs et savoirs-faire différents suivant les acteurs qui y on recours. La grève de la faim de Christian Delorme en 1983 avait constitué un cas typique d'importation de répertoire non violent. Le soutien aux grèves de la part d'associations comme la Cimade se fait souvent au nom de cette non violence. Cette appropriation hétérogène du répertoire de la grève de la faim est telle qu'elle peut aller jusqu'à l'agrégation des catégories du jeûne et de la grève, comme le fit l'abbé Pierre en menant un jeûne au milieu des grévistes déboutés du droit d'asile. Mais malgré cette "importation", la pratique de la grève de la faim montre aussi les caractéristiques d'une somme de savoirs-faire accumulés qui contribuerait presque à lui donner les caractéristiques d'un répertoire autonome: au point, on l'a vu, qu'il s'agisse du répertoire dominant des sans-papiers en France. La recherche du soutien des non violents est alors principalement du domaine de l'opportunité. Par sa rhétorique, par une référence permanente à la violence subie et peut-être à commettre, la grève de la double peine se distinguait nettement du discours non-violent d'un Delorme, comme s'il avait fallu trouver une manière violente de faire une grève de la faim afin que cette dernière "colle" mieux aux propriétés des acteurs qui y avaient recours. S'il fallait évoquer d'autres filiations possibles, outre la référence permanente à l'univers carcéral comme lieu d'apprentissage de la grève de la faim, comme moyen de lutte contre les expulsions, peut être ne serait pas indifférent de se souvenir de la façon dont, de 1957 à 1961, il y eut en France une augmentation considérable du recours à la grève de la faim, suite à la victoire de milliers de détenus politiques algériens du FLN à la prison de Fresnes, lesquels, à l'issue de deux grèves de la faim très sévères, avaient fini par obtenir un régime spécial. De même, la grève des Kurdes et Turcs déboutés se plaçait dans la filiation des nombreuses grèves menées dans les prisons turques ${ }^{54}$. La maîtrise de la grève de la faim par les réfugiés turcs-kurdes était telle que ce sont eux, la plupart du temps, qui ont instauré la pratique de la grève au thé sucré, faisant bénéficier les autres grévistes de cette technique qu'ils justifiaient par l'affirmation qu'elle ne "cassait pas la grève". Dans la grève de la faim de la double peine, c'est une interview d'Abraham Serfaty ${ }^{55}$ dans le journal de la grève qui justifie le recours à "la grève au sucre" et se pose en conseiller ès techniques de grève de la faim. La violence subie qui est au principe de la grève de la faim ne constitue pas seulement des traces objectives que sociologues et médecins seraient supposés mettre à jour : une telle démarche ferait peu de cas de ce qui est considéré comme violence par les sujets qui en furent, ou en sont, l'objet. Alors même qu'il pourrait y avoir violence contre les autres au nom de la violence subie, il semble que ce soit exactement sur la même filiation que repose le recours à la violence contre soi, comme s'il fallait trouver autant 
de raisons de se faire violence que de faire violence aux autres. Si l'on songe à l'énormité de l'affirmation de soi (une "variation de dimension extraordinaire", écrit Maryvonne David Jougneau ${ }^{56}$ ) que représente la menace de se laisser mourir de faim publiquement, il semble qu'il faille une bien puissante justification, un bien fort sentiment de son bon droit pour ne pas apparaître, à soi-même et aux autres, comme assez imbu de soi pour risquer sa vie en croyant qu'elle ait un poids. Ainsi, la grève de la faim est un répertoire de protestation constamment balancé entre autonomie et hétéronomie: souvent seul moyen disponible, elle repose sur une rhétorique profondément misérabiliste ${ }^{57}$ qui mobilise les violences passées au principe de la violence présente. La façon même dont sont réactivés des savoirs accumulés dans la grève de la faim suffit à montrer l'hétéronomie qui est au principe des grèves de sanspapiers. La grève de la faim, répertoire de retournement de stigmates, de retournement de violence symbolique et matérielle, met par exemple en jeu les rares choses que l'on ait appris à faire en prison. Elle oblige à se montrer encore plus misérable qu'on ne le voudrait. Elle ne permet pas toujours de choisir ses soutiens - parfois il semble même que l'on s'en passerait volontiers tant il s'agit de les convaincre de sa propre misère, et donc d'avoir une posture misérabiliste. Par ailleurs, quand la grève de la faim était un moyen que l'on avait pratiqué ailleurs, comme ce fut le cas pour de nombreux déboutés, il avait ici comme un goût dévalué des anciennes luttes - "on ne meurt pas pour des papiers" comme le disait un gréviste débouté -, et nombreux furent les grévistes qui disaient effectivement "avoir honte" de faire grève pour des papiers, n'étant pas loin de souscrire à ce que leur reprochaient les partis kurdes et turcs sur la nécessité d'un engagement "au pays". Car il faut montrer sans cesse sa misère, la domination dont on est l'objet. Ainsi, on exhibe la violence qu'on a subie: tortures, humiliations, incompréhension, expulsions. Les grévistes doivent prouver combien ils sont les souffre-douleur, les victimes de la violence de cette société. Considérée de la sorte, la grève de la faim consiste en une mise en scène théâtralisée des rapports de domination et de violence ("Voyez ces corps que l'Etat fait souffrir"), elle permet de dénoncer une violence de l'Etat qui est dans le prolongement de ce que ce dernier est accusé d'avoir toujours commis. Certains déboutés firent ainsi référence aux souffrances passées en les liant aux souffrances présentes, comparant les pouvoirs turcs et français: "On part, on fuit la répression et ici c'est pareil. Qu'est-ce qu'il attendent pour amener les gaz et faire comme les nazis ils n'ont qu'à amener les gaz ?" ${ }^{58}$. Ce retournement fut particulièrement frappant dans le cas de la grève de la faim contre la double peine, dont toute la rhétorique reposait sur la dénonciation des nombreuses violences qu'avaient subies, selon eux, les jeunes expulsables et au delà, les jeunes immigrés en général. Ainsi la double peine était présentée comme une "violence car elle brise les familles", l'expulsion dénoncée comme une "violence organisée", la prison doublée de l'expulsion comme un "ratonnade légalisée" 59 . On dénonçait de même l'arbitraire des juges qui expulsent, la violence raciste des Français moyens qui assassinent de jeunes Arabes: "C'est la double peine. Cette situation entraîne des divorces, des suicides, le déchirement familial. [...] Nous subissons l'insécurité sociale, nous subissons l'injustice devant les juges. Nous subissons ceux qui tuent les jeunes et moins jeunes"60. Jeudi 30 janvier 1992. Arrive à la table de la conférence de presse Nordine, du Comité contre la double peine et de Résistance des Banlieues. Il dit qu'il est né à Nanterre, qu'il "vaut mieux se faire violence, du moins jusqu'à un certain point", que c'est être normal que de vouloir rentrer dans sa famille quand on a été expulsé [...]. Il évoque des descentes de policiers cagoulés dans les HLM, la violence permanente 
dont les habitants des banlieues seraient l'objet. "On veut une vie normale. [...] Depuis que je suis né je vois que la merde, les $\mathrm{DSQ}^{61}$.....] Au delà de la violence y'a moyen de se faire violence à soi-même". Une autre référence fondatrice à la violence subie est la date du 17 octobre 1961, où des dizaines d'Algériens manifestant à Paris furent tués par la police française. Cette dénonciation effectuée, il est alors rappelé que les grévistes pourraient eux aussi avoir recours à la violence, que cette dernière serait profondément légitime compte tenu de ce qu'ils ont subi. Souvent même ce discours englobe le fait que les grévistes aient été parfois "obligés" d'avoir recours à la violence :"Quand on est clandestin ont est poussé à faire de la violence : on se balade en taxi pour pas se faire arrêter, on paie toujours les tickets de métro pour pas se faire contrôler, mais l'argent il tombe pas du ciel pour tout ça! " ${ }^{62}$. On ne considérer le recours à la grève de la faim comme un avatar des pratiques non violentes, bien au contraire il semble qu'il faille ici l'inscrire dans une véritable grammaire de la violence politique, de la violence incorporée, par des "victimes" qui loin de riposter "se torturent" encore elles-mêmes, accroissant ainsi leur vertu et devenant des malheureux exemplaires. Dire : "Osez donc nous faire violence, nous qui nous faisons déjà souffrir et vous précédons", c'est tenter de renverser un ordre des violences où les victimes revendiquent la primauté du marquage qu'elle s'imposent sur celui qu'elles ont subi. Mais pour ceux qui sont privés de corps politique, c'est encore et toujours démontrer, avec l'éclat de la protestation publique, qu'il n'est d'autre langage de protestation que celui qui consiste à se faire violence, à faire violence à des corps devenus l'ultime refuge d'une protestation qui redouble ainsi leur réduction au biologique.

\section{NOTES}

1. Un gréviste de la faim lors de la grève contre la double peine, 16 janvier 1992.

2. Cf. Michel Foucault, Surveiller et punir, Paris, Gallimard, 1975.

3. "Un modèle où l'expérience accumulée d'acteurs s'entrecroise avec les stratégies d'autorités, en rendant un moyen d'actions limitées plus pratique, plus attractif, et plus fréquent que beaucoup d'autres moyens qui pourraient, en principe, servir les mêmes intérêts". Cf. Tilly, Charles, "Les origines du répertoire de l'action collective contemporaine en France et en Grande-Bretagne", XXème siècle, nº 4, octobre 1984, p. 99.

4. Cf. Pierre Favre, (dir), La manifestation, Paris, Presses de la FNSP, 1990.

5. Il existe ainsi un seul ouvrage de science politique consacré à la grève de la faim : La grève de la faim ou le dérèglement du sacré, Paris, Economica, 1984. Signalons également, dans une approche plus psychanalytique, Kostas Nassikas, Oralité et violence : Du cannibalisme aux grèves de la faim, Paris, L'Harmattan, 1990. Et enfin, Susie Orbach, Hunger Strike : the anorectic's struggle as a metaphor for our age, New York, Norton, 1986. On trouve une littérature plus fournie en ce qui concerne les grèves irlandaises, ainsi que des thèses de médecine et de criminologie. 
6. Cf. Charles Tilly, "Les origines du répertoire de l'action collective contemporaine en France et en Grande-Bretagne", op. cit., p. 89.

7. Comme le soulignent Luc Boltanski, Yann Darre, et Marie-Ange Schiltz, "La dénonciation", Actes de la Recherche en Sciences Sociales, mars 1984, pages. 3-40.

8. Comme le rappelle Michel Dobry, in La Manifestation, op. cit., p. 363.

9. D'un point de vue ethnographique, nous nous baserons ici sur un travail de terrain mené sur deux longues grèves de la faim qui se sont déroulées à Paris début 1992 : celle des 83 déboutés du droit d'asile dans la salle paroissiale de l'Eglise Saint Joseph des Nations (17 janvier / 23 mars), et celle des 25 personnes frappées par la double peine dans des locaux de la Cimade (2 janvier / 17 février).

10. Office Français de Protection des Réfugiés et Apatrides. C'est l'organisme qui décide de l'attribution ou non du statut de réfugié.

11. Entretien auprès d'un gréviste, deux mois après la grève.

12. Répartition des grèves en fonction de leur longueur : plus de 60 jours (1), de 50 à 59 (3), de 40 à 49 (6), de 30 à 39 (10), de 20 à 29 (9), de 10 à 19 (8), de 1 à 9 (9). Soit 685 personnes ayant mené des grèves de 30 jours à plus de 60 . (Source : coordination nationale de soutien aux déboutés du droit d'asile)

13. Ce journal, dont il sortit trois numéros, fut édité par l'agence Im'média.

14. De 19 au 2 janvier, il passa à 27 au 8, 18 au 18, 21 au 20, 19 fin janvier, 17 au 7 février. Comptage réalisé à partir des communiqués du comité et de la Cimade.

15. A cet égard, la parution pendant la grève de la double peine d'une affiche représentant un groupe de grévistes dont certains avaient au moment de la photographie déjà arrêté la grève n'est pas sans rappeler la fonction symbolique qu'eut la référence aux "Dix" de Billancourt, ces "Dix" qui ne furent pas les mêmes tout au long du conflit. Cf. Virginie Linhart, "Les "Dix" de Renault-Billancourt. Les enjeux d'une mobilisation d'appareil", Revue Française de science politique, Vol. 42, n³, juin 1992,

pp. 375-401.

16. Thomas Schelling, Stratégie du conflit, Paris, PUF, 1986, p. 176.

17. Ici à comprendre comme "un racisme contre le commerçant français".

18. Un membre du comité contre la double peine, gréviste de la faim, lors de la conférence de presse du 21 janvier 1992.

19. Cf. Anthony Oberschall, Social Conflict and Social Movements, Englewood Cliffs, Prentice Hall, 1973.

20. Ainsi les lieux d'accueil des grèves de la faim recensés par la coordination nationale de soutien aux déboutés du droit d'asile se répartissent ainsi : paroisses catholiques, églises ou salles (38), paroisses protestantes (3), locaux associatifs (2), locaux syndicaux (2), local politique (PS) (1), Sans local (3).

21. Entretien auprès d'un des principaux membres de la coordination nationale de soutien aux déboutés, ancien prêtre, membre de la Fasti, vendredi 17 avril 1992.

22. Un responsable associatif lors d'une réunion du comité de soutien aux déboutés, le 12 mars 1992.

23. Un membre du comité de soutien aux déboutés, réunion du 12 mars 1992.

24. Notes de terrain. Conférence de presse au cours de la grève de la faim des personnes touchées par la double peine, jeudi 30 janvier 1992.

25. Entretien 13 août 1992.

26. Devant le refus des médecins, ce fut finalement un capitaine des pompiers, envoyé par la préfecture, qui vint faire une visite de quelques minutes à Saint Joseph.

27. Ce fut notamment le cas dans les grèves d'Orléans et de Bordeaux. 
28. Ou plutôt qu'on ne laisse pas quelqu'un mourir de grève de la faim en France, l'apparition d'un coma aboutissant systématiquement à une hospitalisation dont les militants associatifs prennent la responsabilité.

29. Au bout d'un mois de grève sans vitamines sont susceptibles d'apparaître des séquelles irréversibles, les cellules nerveuses étant les seules à ne pas se renouveler : d'où troubles de mémoire, altération définitive de la motricité et de la vue, etc.

30. Ainsi une insuffisance rénale grave, une pneumonie, une femme enceinte (il y avait 7 femmes sur la grève des déboutés), un doigt cassé, une insuffisance cardiaque...

31. Cas de figure évoqué par Thomas Schelling dans Stratégie du conflit, op. cit.., page 35 et suivantes.

32. Un gréviste turc, traduit, s'adressant aux membres du comité de soutien, réunion du 19 mars 1992.

33. Cf. Richard Kearney, "Terrorisme et sacrifice. Le cas de l'Irlande du Nord", Esprit, avril 1979. p. 32.

34. Sur les suffragettes anglaises, les premiers grévistes irlandais, des dissidents soviétiques, des grévistes Irlandaises comme les soeurs Maleor et Dolores Price qui restèrent 200 jours sous perfusion, etc.

35. D'où la pratique courante dans les grèves de la signature d'une décharge : ainsi, à Bordeaux, "je déclare posséder mes possibilités habituelles de réflexion et de décision ; et donc en toute conscience refuser l'hospitalisation forcée qui est ordonnée à mon encontre."

36. Cf. Georges Balandier, "Le politique des anthropologues", in Madeleine Grawitz, Jean Leca, Traité de science politique, t. 1. Paris, PUF., 1985. p. 322.

37. Avec une exception notable qui se situa significativement au coeur du mouvement des grèves de la faim : la manifestation en plein Paris d'une dizaine de milliers de clandestins, le 25 mai 1991.

38. N'étant pas des nationaux voire des étrangers en situation régulière.

39. Menaces physiques, racket, meurtres...

40. Sur le retournement des stigmates transformés en principes d'action et de légitimité, voir Erving Goffman, Stigmate. Les usages sociaux des handicaps, Paris, Editions de Minuit, 1989. p. 122, et Howard Becker, Outsiders. Etudes de sociologie de la déviance, Paris, Métailié, 1990.

41. Sur le panoptique de Bentham, voir Michel Foucault, Surveiller et punir, op. cit., page 201 et suivantes.

42. Rappelons que la plupart des expulsions rattachées à la double peine opèrent dans le cadre de l'article 26 du code pénal, qui prévoit l'expulsion notamment dans le cas de trafic de stupéfiants, notion interprétée assez extensivement par les juges (cent grammes de haschich suffisent à entrer dans cette catégorie) pour peu que le coupable soit étranger et de surcroît qu'il fasse appel d'une condamnation à ce sujet.

43. Un gréviste de la double peine. Entretien du 21 mai 1992.

44. Sur cette notion, voir Erving Goffman, Stigmate. Les usages sociaux des handicaps, op. cit., p. 224-225.

45. Entretien lors de la grève de la double peine, 16 janvier 1992.

46. "Ainsi dissocié de tout ordre national, celui de l'immigration et celui de l'émigration, (...) l'immigré pourrait finir, comme finit par exemple le déporté, par n'être plus rien : rien d'autre qu'une identité singulière et corporelle, un corps biologique et technique, "un corps labeur" ; Abdelmalek Sayad, L'immigration ou les paradoxes de l'altérité. Bruxelles, De Boeck/Paris : Editions Universitaires, 1991, p. 298. 
47. Un gréviste de la double peine. Entretien du 21 mai 1992.

48. La métaphore corporelle étant à ce point puissante qu'elle permet de dire que l'étranger est expulsé par la force, comme un corps étranger au corps politique.

49. Nous serions ici tentés de faire référence à l'oeuvre de Kantorowicz si la problématique des "deux corps du roi", la division entre son corps physique et périssable et son corps politique, immortel, n'était comme son auteur l'indique une construction symbolique historiquement située et limitée. Pourtant la distinction entre corps politique et corps physique parait ici particulièrement heuristique. Il y aurait ainsi deux corps du sujet politique, le premier étant au principe de la reconquête du second. Cf. Ernst Kantorowicz, Les deux corps du roi. Essai sur la théologie politique au Moyen Age, Paris, Gallimard NRF, 1989.

50. Un gréviste de la double peine. Entretien du 21 mai 1992.

51. Ce retournement allant jusqu'à l'étonnement devant l'estime voire simplement l'attention dont ils jouissaient tout à coup, attention que certains jugeaient décalée par rapport à l'image qu'ils avaient d'eux mêmes. Ainsi ce gréviste déclarant : "T'es cool. T'as du courage de te balader au milieu de nous" "Pourquoi ?" "Ben on est quand même des délinquants, non ?".

52. En ce qui concerne ces dimensions de l'estime de soi et de la grandeur de soi, l'ouvrage de Maryvonne David-Jougneau, Le dissident et l'institution ou Alice au pays des normes, Paris, L'Harmattan, 1989, est d'un grand intérêt. S'appuyant sur une interprétation sociologique et psychanalytique d'Alice au pays des Merveilles, l'auteur souligne comment l'affirmation de soi et de son sens de la justice semble corollaire d'un changement de dimension du soi. "En accédant à sa full size, en ayant osé s'affirmer et affirmer ses valeurs, Alice a réduit, tels des baudruches, le pouvoir et la dimension fantasmés de tous ces adultes qui détiennent un pouvoir institué dont ils abusent, et qui vont se trouver réduits à leurs fonctions devenus simples cartes à jouer... Pour opérer cette réduction (...) il suffit de s'affirmer comme un être réel qui ose faire front à un "ordre" qu'il perçoit comme désordre, ou à une "loi" qui semble l'expression de l'arbitraire.", p. 193.

53. Hocine, gréviste, lors d'une réunion qui marquait la fin de la grève de la double peine.

54. Selon L'Humanité du 25 mai 1990, le 24 mai 1990 aurait commencé une grève de 500 détenus politiques dans 5 prisons turques, en solidarité avec 10 autres prisonniers dont 8 journalistes qui jeûnaient afin de protester contre un article du code pénal limitant la liberté d'expression.

55. Ancien prisonnier politique marocain, il mena plusieurs grèves de la faim.

56. Maryvonne David-Jougneau, op. cit.

57. Au sens où l'entendent Claude Grignon et Jean-Claude Passeron dans Le savant et le populaire. Misérabilisme et populisme en sociologie et en littérature, Paris, Gallimard/ Le Seuil, Hautes Etudes, 1989. Sur l'application de cette problématique à cette catégorie de dominés que sont les immigrés, voir Catherine Polac, Quand les immigrés prennent la parole. Histoire sociale du journal Sans Frontières. 1979-1985. Mémoire présenté pour le DEA d'Etudes Politiques de Paris, sous la direction de Jean Leca, 1991.

58. Des déboutés aux membres du comité de soutien, jeudi 19 mars 1992.

59. Un tract de la grève de la double peine, janvier 1992.

60. Tract de Résistance des banlieues, février 1992.

61. Programme sociaux

62. Conférence de presse du 30 janvier 1992. 


\section{RÉSUMÉS}

Les deux plus grandes vagues de grèves de la faim ayant eu lieu, en France, hors milieu carcéral furent menées par des sans-papiers, ce qui fait de la grève de la faim le répertoire essentiel de ce groupe social. Considérer ce répertoire à partir des catégories de la violence politique revient à souligner l'intérêt que représentent les phénomènes de "violence contre soi" rapportés à la problématique du marquage des corps, à celle des "technologies politiques du corps", envisagées non plus du point de vue d'une entité politique dominante mais plutôt sous l'angle du sujet politique. Cela permet de ne pas se limiter à l'étude dimension instrumentales du "procédé" : le répertoire suppose un univers de rareté des moyens, mais aussi un univers de sens partagé. Sous la dénomination "grève de la faim" se cachent ainsi de multiples façons de se "faire violence" au nom de violence déjà subies. Un éclairage du recours à la grève de la faim être fourni ici à partir des parcours des populations concernées, comme si le recours à la grève ne faisait sens qu'en s'insérant dans une anthropologie politique des corps touchant à la fois à la question du monopole de la violence légitime et aux dimensions symboliques de la violence physique incorporée.

\section{INDEX}

Mots-clés : étrangers, immigration, pauvreté 